\title{
Modulation of unsaturated fatty acids content in algae Spirulina platensis and Chlorella minutissima in response to herbicide SAN 9785
}

\author{
Deepa Kachroo \\ Microbiology Division \\ Centre for Biochemical Technology \\ Delhi University Campus \\ Mall Road, New Delhi, India \\ Shaneen M. Singh Jolly \\ Department of Biology \\ Brooklyn College \\ City University of New York \\ 2900 Bedford Avenue \\ Brooklyn NY 11210, USA \\ Tel: 17189515720 \\ E-mail: ssingh@brooklyn.cuny.edu \\ Viraraghavan Ramamurthy* \\ Department of Biotechnology \\ PSG College of Technology \\ Coimbatore 641004 India \\ Tel: 914224344777 \\ Fax: 914222573833 \\ E-mail: ram@email.psgtech.ac.in
}

Keywords: Chlorella minutissima, fatty acid desaturation, polyunsaturated fatty acids, Spirulina platensis, SAN 9785.

Abbreviations: GLA: $\gamma$-linolenic acid

PUFA: polyunsaturated fatty acids

The accumulation of polyunsaturated fatty acids by algae Spirulina platensis and Chlorella minutissima was studied. Response of these organisms to the substituted pyridazinone, SAN 9785, an inhibitor of the long chain fatty acid desaturase, indicated that fatty acid synthesis and their desaturation were regulated differently in these organisms. While the pool of palmitic acid, the precursor for the unsaturated $\mathrm{C}_{18}$ fatty acids, was stringently maintained in the green alga $C$. minutissima, in the cyanobacterium $S$. platensis the level of palmitic acid was liberally maintained in spite of the enhanced accumulation of unsaturated $C_{18}$ fatty acids.

The health benefits of polyunsaturated fatty acids (PUFA) has spurred interest in their commercial production. Microalgae have been an attractive source of PUFA (Benemann et al. 1987) due to their inherently high PUFA content, de novo biosynthetic capabilities, amenability to genetic manipulation, as well as to the employability of biochemical selection strategies, and the possibility of cultivation to high densities in limited space. Several algae, both prokaryotic and eukaryotic, have inherently high amounts of PUFA, and further respond to stress conditions by altering their PUFA concentration. Cyanobacterium
Spirulina is rich in $\gamma$-linolenic acid (GLA) (and poor in the $\alpha$-isomer) and thus is a good source for the purification of this PUFA (Mahajan and Kamat, 1995). Chlorella minutissima is an eukaryotic alga, with a fast growth rate and high PUFA content (Seto et al. 1984) and could be another important source of a PUFA-rich nutraceutical supplement. Since these algae could be relatively easily cultivated at different stress conditions, they offer the prospect of a good source of PUFA for the nutraceutical market.

Murphy et al. had shown that the substituted pyridazinone SAN 9785 inhibits the desaturation of long chain fatty acids (Murphy et al. 1985), and this finding has been used for obtaining strains overproducing PUFA (Cohen et al. 1993). We have compared the response of Spriulina platensis and C. minutissima, to SAN 9785 to study the mechanisms behind overproduction of unsaturated fatty acids in these organisms.

\section{MATERIALS AND METHODS}

\section{Culture conditions}

*Corresponding author 
Table 1. Effect of SAN 9785 on the fatty acid profile of S. platensis.

\begin{tabular}{|c|c|c|c|c|c|c|}
\hline \multicolumn{2}{|c|}{ Conc. (mM)* } & Palmitic acid & Stearic acid & Oleic acid & Linoleic acid & Linolenic acid \\
\hline Stock & Transfer & C16:0 & C18:0 & C18:1 & C18:2 & C18:3 \\
\hline 0 & 0 & $6685 \pm 1338$ & ND & $46 \pm 20$ & $1060 \pm 340$ & $726 \pm 24$ \\
\hline 0 & 0.2 & $5783 \pm 980$ & ND & $18 \pm 11$ & $407 \pm 67$ & $120 \pm 38$ \\
\hline 0.2 & 0.2 & $3534 \pm 145$ & $589 \pm 38$ & $2390 \pm 98$ & $2192 \pm 237$ & $3783 \pm 549$ \\
\hline 0.2 & 0 & $5358 \pm 272$ & $138 \pm 19$ & $1359 \pm 199$ & $1334 \pm 105$ & $1653 \pm 88$ \\
\hline
\end{tabular}

*Independent stock cultures were maintained at the indicated concentration of SAN 9785 in quadruplicate. For the fatty acid profile determination the cultures were transferred to medium containing the indicated concentration of SAN 9785, harvested after ten days and assayed. Fatty acid concentration is indicated as ug per g dry biomass \pm standard error of the mean. ND: not detectable.

Spirulina platensis was obtained from Ballarpur Industries Limited, New Delhi, and Chlorella minutissima from Sammlung von Algenkulturen, Göttingen. SAN 9785, developed by BASF, was obtained from the laboratory of Dr. J. St. John, US Department of Agriculture, Beltsville, MD.

S. platensis was grown in Zarrouk medium (Starr and Zeikus, 1993) at $28^{\circ} \mathrm{C}$ under cyclic fluorescent illumination (14 hrs light: $10 \mathrm{hrs}$ dark; 3000 lux). C. minutissima was grown in artificial sea water medium (Starr and Zeikus, 1993) in a glass house maintained at $32^{\circ} \mathrm{C}$, illuminated by indirect sunlight. To the culture medium SAN 9785 stock prepared in sterile DMSO was added as per the experimental design and inoculated with exponentially growing cultures. Tolerant cultures were obtained by repeated subculture at $4 \mathrm{~d}$ interval in growth medium supplemented with SAN 9785 at $0.2 \mathrm{mM}$ concentration. Stable tolerant cultures of both the algae were obtained in 2 months.

\section{Analytical procedures}

Biomass concentration of $S$. platensis was estimated by measuring the optical density (OD) at $560 \mathrm{~nm}$ of appropriately diluted cultures. Cell density of $C$. minutissima was counted using a hemocytometer. Both OD and cell number measurements were caliberated with dry weight estimations of several culture samples. Lipids were extracted from exponentially growing cultures in chloroform methanol mixture $(2: 1 \mathrm{v} / \mathrm{v})$, at $28^{\circ} \mathrm{C}$ for $24 \mathrm{hrs}$ in darkness and filtered. The extract was mixed thoroughly with half volume of $0.9 \% \mathrm{NaCl}$ solution and the organic phase containing free fatty acids was separated (Jayaraman, 1981). The solvent evaporated under nitrogen and the lipid content was gravimetrically estimated. Fatty acid composition of extracted lipid was determined after transmethylation with methanol-acetyl chloride (Cohen and
Cohen, 1991). Gas chromatographic analysis was carried out using a DEGS column $(30 \mathrm{~m} \times 0.38 \mathrm{~mm}$, Nucon Instruments, New Delhi) maintained at $190^{\circ} \mathrm{C}$ and $\mathrm{N}_{2}$ as the carrier gas at the rate of $30 \mathrm{ml} / \mathrm{min}$. The injector and detector ports were maintained at $220^{\circ} \mathrm{C}$. Peaks were identified using an FID detector by comparison with reference standards (Sigma Chemical Co., St. Louis, MO.) and area measured using an integrator. The data shown are the mean of four independent replicate cultures.

\section{RESULTS AND DISCUSSION}

\section{Effect of SAN 9785 on the growth of the algae}

To evaluate the response of the algae to SAN 9785, cultures of Spirulina platensis and Chlorella minutissima were grown in media containing different concentration of SAN 9785, and the concentration of biomass as a function of time was monitored (Figure 1). S. platensis cells exposed to $0.8 \mathrm{mM}$ SAN 9785 were completely lyzed within a week. Cells exposed to $0.2 \mathrm{mM}$ SAN 9785 exhibited a prolonged lag phase before increase in cell mass could be observed, while cells exposed to $0.4 \mathrm{mM}$ SAN 9785 were static for more than one month. The surviving cells exhibited exponential growth, which however was significantly lower than that in cultures not exposed to the herbicide. Although the cells that were growing had acclimatized to the lower herbicide concentration, they did not exhibit the growth rate observed under the herbicide-free condition. This is in contrast to the observation of Cohen et al. (1993), who report an enhanced growth rate in herbicide resistant $S$. platensis isolates. The reduced growth rate suggested that the herbicide interfered with metabolic activities required for normal growth rate, and the herbicide itself was not inactivated under the prevailing conditions of the culture, like high alkalinity, exposure to light, high dissolved oxygen content, etc. 
Dissimilar response to herbicide SAN 9785 by Spirulina and Chlorella

Table 2. Effect of SAN 9785 on the fatty acid profile of $C$. minutissima.

\begin{tabular}{|c|c|c|c|c|c|c|}
\hline \multicolumn{2}{|c|}{ Conc. (mM)* } & Palmitic acid & Stearic acid & Oleic acid & Linoleic acid & Linolenic acid \\
\hline Stock & Transfer & C16:0 & C18:0 & C18:1 & C18:2 & C18:3 \\
\hline 0 & 0 & $7126 \pm 624$ & $27 \pm 13$ & $932 \pm 275$ & $2316 \pm 640$ & $1166 \pm 359$ \\
\hline 0 & 0.2 & $6030 \pm 458$ & $35 \pm 16$ & $348 \pm 58$ & $212 \pm 57$ & $297 \pm 58$ \\
\hline 0.2 & 0.2 & $7158 \pm 866$ & $34 \pm 14$ & $407 \pm 90$ & $1712 \pm 340$ & $5426 \pm 362$ \\
\hline 0.2 & 0 & $8305 \pm 586$ & $22 \pm 14$ & $4042 \pm 195$ & $6380 \pm 435$ & $1971 \pm 83$ \\
\hline
\end{tabular}

${ }^{*}$ Conditions of culture maintenance and analysis same as that of S. platensis, described in Table 1.

C. minutissima was generally more tolerant to SAN 9785 as compared to $S$. platensis. At the highest concentration examined, C. minutissima cells were substantially lyzed, and the remaining cells did not grow any further in 10 weeks. However, at lower concentrations of SAN $9785(0.2$ and $0.4 \mathrm{mM}$ ), the growth rate was suppressed, and the level of suppression was proportional to the concentration of SAN 9785 in the culture. However, C. minutissima cells acclimatized to SAN 9785 more readily, since at $0.2 \mathrm{mM}$ concentration the growth rate suppression was only marginal compared to control culture, while in S. platensis the difference in growth rate was substantial. This suggested that the metabolic processes affected by SAN 9785 was less influential on the overall growth of $C$. minutissima, as compared with the case in S. platnensis.

The lipid content of the cells tolerant to SAN 9785 in both the algae did not show significant difference. In S. platensis cultures the lipid content was $7.5 \pm 0.2 \%$ of the dry weight in both the control as well as tolerant cultures. Similarly in C. minutissima the lipid content was $7.1 \pm 0.3 \%$ of the dry weight in both the control and tolerant cultures. Otles and Pire (2001) report similar value for $S$. platensis, but much higher value for other Chlorella species (C. pyrenoidosa and $C$. vulgaris). The variability in the lipid content observed in both the algae in our study was more influenced by the environmental and growth conditions rather than by their response to the herbicide (data not shown).

\section{Effect of SAN 9785 on the free fatty acid composition of S. platensis}

In S. platensis growing in $0.2 \mathrm{mM}$ SAN 9785 the amount of palmitic acid (16:0) decreased gradually and stabilized at around $52 \%$ of the palmitic acid content observed in control culture. This reduced amount was maintained as long as the cultures were exposed to SAN 9785. Upon removal of the herbicide however, palmitic acid content began to increase to a concentration as high as observed in cultures not exposed to the herbicide at all. In contrast, stearic acid (saturated fatty acid, 18:0) which was not detectable in control culture rose to detectable level in cells exposed to and surviving in the presence of SAN 9785. The amount of the $\mathrm{C}_{18}$ unsaturated fatty acids - oleic (18:1), linoleic (18:2) and linolenic (18:3) acids - initially decreased when the cultures were exposed to SAN 9785. Upon extended cultivation in the presence of the herbicide the amount of these unsaturated fatty acids increased and became a substantial fraction of the free fatty acids. However, this enhanced amount of the unsaturated fatty acids was detectable only as long as the cultures were grown in the presence of the herbicide. Upon removal of the herbicide, these unsaturated fatty acids and their presumed immediate saturated precursor, stearic acid, decreased while the content of the $\mathrm{C}_{16}$ fatty acid palmitic acid increased, approaching a profile seen in cultures not exposed to the herbicide.

\section{Effect of SAN 9785 on the free fatty acid composition of C. minutissima}

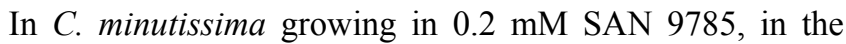
first ten days the amount of palmitic acid decreased by about $15 \%$, but upon continuous exposure to the herbicide it reached a level as high as that observed in control culture. Upon removal of the herbicide the palmitic acid content increased by about $15 \%$ in ten days, compared to its level in untreated culture. In contrast to this, the stearic acid content did not show much variation and was observed to be at a low concentration in the cultures irrespective of their SAN 9785 exposure status. The amount of the unsaturated fatty acids, oleic, linoleic and linolenic acids initially decreased by $62 \%, 91 \%$ and $75 \%$, respectively, when the culture was exposed to SAN 9785. But upon extended growth in the presence of the herbicide, the concentration of these acids showed substantial recovery. While the amount of palmitic, stearic, oleic and linoleic acids was comparable to control culture, the amount of linolenic acid, in contrast, after an initial decrease upon exposure to $0.2 \mathrm{mM}$ SAN 9785, 

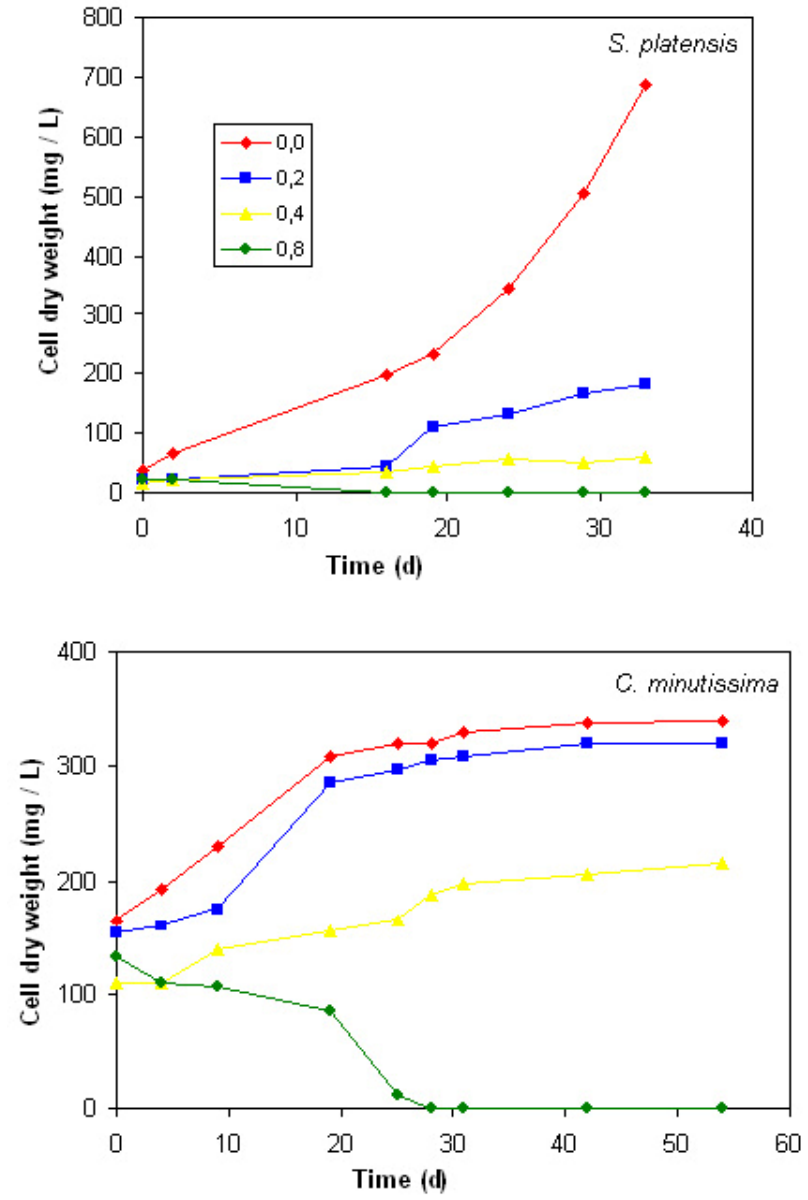

Figure 1. Effect of SAN-9785 on the growth of S. platensis and C. minutissima. Biomass of cultures growing in different concentration (mM) of SAN 9785 is shown as a function of time.

increased to an amount almost five times to that found in control culture. However, upon removal of the herbicide, the amount of oleic and linoleic acids accumulated to a concentration higher than that found in control culture while the elevated amount of linolenic acid was reduced to a concentration closer to that found in control culture. Thus, in the presence of fatty acid desaturase inhibitor, the tolerant cells of both $S$. platensis and C. minutissima overcompensate with enhanced accumulation of linolenic acid.

These results indicate the difference in the regulation of fatty acid synthesis but not the desaturase activity between $S$. platensis and C. minutissima. In both the algae, response to continuous exposure to SAN 9785 was by elevating their linolenic acid content. It is likely that prolonged subculture in $0.2 \mathrm{mM}$ SAN 9785 had selected for cells with enhanced fatty acid desaturation efficiency whereby the level of desaturated free fatty acid was elevated. Although other investigators have suggested that SAN 9785 effects could be pleiotropic in eucaryotic algae and some higher plants like maize (Murphy et al. 1985) and eustigmatophyte (Khozin and Cohen, 1996), the organisms studied in this report showed similar response as far as linolenic acid level enhancement was concerned. However, the fate of its precursors in the algae differed. While in S. platensis there appeared to be no compensation of palmitic acid while its utilization for the synthesis of the unsaturated fatty acids had increased upon exposure of the culture to SAN 9785, in C. minutissima the increased consumption of the saturated fatty acids, as a consequence of increased conversion to unsaturated fatty acids was compensated. This suggests that in C. minutissima there was an increased de novo fatty acid synthesis. While in C. minutissima the amount of saturated fatty acids, palmitic and stearic acid, were stringently maintained at amounts observed in untreated cultures, in $S$. platensis their amounts were greatly influenced by their conversion to the desaturated fatty acids. Further, in $S$. platensis, similar trend of accumulation of the different unsaturated fatty acids suggests that the enzymes involved in the individual step of the desaturation reactions had similar response to SAN 9785. The three genes involved in desaturase activity in $S$. platensis have different specificity (Apiradee et al. 2004), but may respond to SAN 9785 similarly. In the case of $C$. minutissima, it is not clear whether different enzymes are used or the same enzyme carries out the succeeding steps of desaturation. Thus, the non-uniform change in the amount of different unsaturated fatty acids in C. minutissima suggests that the influence of the herbicide on each step of reduction, or the activity of the different desaturating enzymes for the succeeding steps of the reaction was likely to be different. It is suggested that the C. minutissima desaturases are more similar to the multiple fatty acid desaturases identified in plants (Sprecher et al. 1995; Heppard et al. 1996). Also, it is likely that $C$. minutissima possesses both chloroplastic as well as cytoplastic fatty acid metabolisms, responding to SAN 9785 differently, as suggested by Khozin and Cohen (1996).

\section{CONCLUDING REMARKS}

These studies suggest that $S$. platensis responds to SAN 9785 by driving the fatty acid desaturation pathway without compensating for the enhanced conversion of the precursor palmitic acid, while $C$. minutissima responds by adequate compensation of the fatty acid precursor pool, possibly through enhanced de novo synthesis. Perhaps due to this robustness of $C$. minutissima, it was able to tolerate higher doses of SAN 9785.

\section{ACKNOWLEDGMENTS}

The authors thank Dr. MP Kapoor, Director, Thapar Institute of Engineering and Technology, Patiala for providing infrastructure facilities and Dr. H.A. Norman and Dr. J. St. John of USDA, Beltsville, MD, for the generous gift of SAN 9785 used in these studies. SJ acknowledges the Research Fellowship provided by the Council of Scientific and Industrial Research, New Delhi. 


\section{REFERENCES}

APIRADEE, Hongsthong;

KALYANEE, Paithoonrangsarid; PONGSATHON, Prapugrangkul; PATCHARAPORN, Deshnium; MATURA, Sirijuntarut; SANJUKTA, Subhudhi; SUPAPON, Cheevadhanarak and MORAKOT, Tanticharoen. The expression of three desaturase genes of Spirulina platensis in Escherichia coli DH5a - heterologous expression of Spirulina-desaturase genes. Molecular Biology Reports, September 2004, vol. 31, no. 3, p. 177-189.

BENEMANN, John R.; TILLETT, David M. and WEISSMAN, Joseph C. Microalgae biotechnology. Trends in Biotechnology, February 1987, vol. 5, no. 2, p. 47-53.

COHEN, Zvi and COHEN, Simon. Preparation of eicosapentaenoic acid (EPA) concentrate from Porphyridium cruentum. Journal of the American Oil Chemists Society, January 1991, vol. 68, no. 1, p. 16-19.

COHEN, Zvi; REUNGJITCHACHAWALI, Marasri; SIANGDUNG, Wipawan; TANTICHAROEN, Morakot and HEIMER, Yair M. Herbicide-resistant lines of microalgae: Growth and fatty acid composition. Phytochemistry, November 1993, vol. 34, no. 4, p. 973-978.

HEPPARD, E.P.; KENNEY, A.J.; STECCA, K.L. and MIAO, G.H. Developmental and growth temperature regulation of two different microsomal w-6 desaturase genes in soybeans. Plant Physiology, January 1996, vol. 110, no. 1, p. 311-319.

JAYARAMAN, J. Laboratory Manual in Biochemistry. New Delhi, New Age Publishers, 1981, 180 p. ISBN 0852264283.

KHOZIN, Inna and COHEN, Zvi. Differential response of microalgae to the substituted pyridazinone, sandoz 9785 , reveal different pathways the biosynthesis of eicosapentaenoic acid. Phytochemistry, July 1996, vol. 42, no. 4 , p. $1025-1029$.

MAHAJAN, G. and KAMAT, M. $\gamma$-Linolenic production from Spirulina platensis. Applied Microbiology and Biotechnology, July 1995, vol. 43, no. 3, p. 466-469.

MURPHY, Denis J.; HARWOOD, John N.; LEE, Kevin A.; ROBERTO, Francisco; STUMPF, Paul K. and ST. JOHN, Judith B. Differential responses of a range of photosynthetic tissues to a substituted pyridazinone, Sandoz 9785. Specific effects on fatty acid desaturation. Phytochemistry, September 1985, vol. 24, no. 9, p. 19231929.

OTLES, Semih and PIRE, Ruhşen. Fatty acid composition of Chlorella and Spirulina microalgae species. Journal of AOAC International, November 2001, vol. 84, no. 6, p. 1708-1714.
SETO, Akira; WANG, H.L. and HESSELTINE, C.W. Culture conditions affect eicosapentaenoic acid content of Chlorella minutissima. Journal of the American Oil Chemists Society, May 1984, vol. 61, no. 5, p. 892-894.

SPRECHER, H.; LUTHRIA, D.L.; MOHAMMED, B.S. and BAYKOUSHEVA, S.P. Reevaluation of the pathways for the biosynthesis of polyunsaturated fatty acids. Journal of Lipid Research, September 1995, vol. 36, no. 12, p. 2471-2477.

STARR, Richard C. and ZEIKUS, Jeffrey A. UTEX-The culture collection of algae at the University of Texas at Austin 1993 list of cultures. Journal of Phycology, April 1993, vol. 29, no. 2 (supplement), p. 1-106. 